Y. Hashimoto and T. Matsuzawa

Nagoya Math. J.

Vol. 55 (1974), 181-204

\title{
ON A CLASS OF DEGENERATE ELLIPTIC EQUATIONS
}

\author{
YOSHIAKI HASHIMOTO AND TADATO MATSUZAWA
}

We shall prove in Chapter I the hypoellipticity ${ }^{1)}$ for a class of degenerate elliptic operators of higher order. Chapter II will be devoted to the consideration of the regularity at the boundary for the solutions of general boundary problems for the equations considered in Chapter I being restricted to the second order.

\section{Chapter I. Hypoellipticity for a class of degenerate elliptic operators.}

\section{§ 1. Introduction.}

In [5], Grušin has proved the hypoellipticity for a class of degenerate elliptic equations. Our aim in this chapter is to give a simple proof with some additional assumptions on the operators considered in [5].

First we state the main result obtained in [5]. Let $\boldsymbol{R}^{N}$ be $\mathrm{N}$-dimensional Euclidean space regarded as a direct product of two Euclidean spaces $\boldsymbol{R}^{k}$ and $\boldsymbol{R}^{n}(k+n=N)$. We consider a pair $(\rho, \sigma)$ of $N$ rational numbers $\left(\rho_{1}, \cdots, \rho_{N}\right),\left(\sigma_{1}, \cdots, \sigma_{N}\right)$ such that $\rho_{j} \geqq 1$ and $\sigma_{j} \geqq 0(1 \leqq j \leqq N)$ and that

$$
\text { (a) } \quad \rho_{j}=\sigma_{j}=1 \quad k+1 \leqq j \leqq k+n=N
$$

and for each $j, 1 \leqq j \leqq k$, one of the following conditions is satisfied:

$$
\begin{gathered}
\rho_{j}>\sigma_{j}>0, \\
\sigma_{j}=0 .
\end{gathered}
$$

Suppose $(\rho, \sigma)$ is given. The following notations are convenient for the later discussions:

$$
x=\left(x_{1}, \cdots, x_{N}\right) \in \boldsymbol{R}_{N},
$$

Received April 1, 1974.

1) A differential operator $P$ is said to be hypoelliptic, if any distribution $u$ is infinitely differentiable in every open set where $P u$ is infinitely differentiable. 


$$
\begin{aligned}
x & =\left(x^{\prime}, y\right), x^{\prime}=\left(x_{1}, \cdots, x_{k}\right), y_{j}=x_{k+j} \quad 1 \leqq j \leqq n, \\
x^{\prime} & =\left(x^{\prime \prime}, x^{\prime \prime \prime}\right), x^{\prime \prime}=\left(x_{1}, \cdots, x_{k^{\prime}}\right), x^{\prime \prime \prime}=\left(x_{k^{\prime}+1}, \cdots, x_{k}\right)
\end{aligned}
$$

where $k^{\prime}$ is in agreement with the number of $j$ satisfying (b).

Let $m$ be a positive integer and set

$$
\begin{gathered}
\mathfrak{M}=\{(\gamma, \alpha) ;|\alpha| \leqq m,\langle\rho, \alpha\rangle \geqq\langle\sigma, \gamma\rangle \geqq\langle\rho, \alpha\rangle-m\}, \\
\mathfrak{M}_{0}=\{(\gamma, \alpha) ;|\alpha| \leqq m,\langle\sigma, \gamma\rangle=\langle\rho, \alpha\rangle-m\},
\end{gathered}
$$

where $(\gamma, \alpha)$ is a pair of $N$-tupled multi-indices of non-negative integers and $\gamma_{j}=0$ for $j$ if $\sigma_{j}=0(1 \leqq j \leqq k)$. We use the following notations:

$$
\begin{aligned}
|\alpha| & =\alpha_{1}+\cdots+\alpha_{N}, \\
\langle\rho, \alpha\rangle & =\rho_{1} \alpha_{1}+\cdots+\rho_{N} \alpha_{N} \quad \text { etc } .
\end{aligned}
$$

Now we consider a partial differential operator

$$
L(x, D)=\sum_{(\gamma, \alpha) \in \mathfrak{R}} a_{\alpha_{\gamma}}(x) x^{\gamma} D^{\alpha},
$$

where $a_{\alpha \gamma}(x) \in C^{\infty}\left(\boldsymbol{R}^{N}\right),(\alpha, \gamma) \in \mathfrak{M}$ and

$$
D=\left(\frac{1}{i} \frac{\partial}{\partial x_{1}}, \cdots, \frac{1}{i} \frac{\partial}{\partial x_{N}}\right) .
$$

Associated with (1.3) we consider the partial differential operator with polynomial coefficients :

$$
L_{0}\left(x^{\prime \prime}, y ; D\right)=\sum_{(r, \alpha) \in \mathbb{M}_{0}} a_{\alpha \gamma}(0) x^{r} D^{\alpha} .
$$

Condition 1. $L_{0}\left(x^{\prime \prime}, y ; D\right)$ is elliptic for $\left|x^{\prime \prime}\right|+|y|=1$.

Condition 2. The differential equation

$$
\hat{L}_{0}\left(x^{\prime \prime}, y ; \xi, D_{y}\right) v(y)=\sum_{\substack{\gamma, \alpha) \in \mathfrak{m}_{0} \\ \alpha=\left(\alpha^{\prime}, \beta\right)}} a_{\alpha \gamma}(0) x^{r} \xi^{\alpha^{\prime}} D_{y}^{\beta} v(y)=0
$$

has no non-trivial solution in $\mathscr{S}\left(\boldsymbol{R}_{y}^{n}\right)$ for any fixed $\xi \in \boldsymbol{R}^{k}, \xi \neq 0$, and $x^{\prime \prime}$. Set

$$
\rho_{0}=\min _{1 \leqq j \leqq k} \rho_{j}, \quad \sigma^{0}=\max _{1 \leqq j \leqq k} \sigma_{j} .
$$

Having established these notations the main result of Grušin can be stated as follows:

THEOREM 1.1 ([5, Theorem 1.1]). Assume $\rho_{0}>\sigma^{0}$. Under the con- 
ditions 1 and 2, the operator $L(x, D)$ is hypoelliptic in a neighborhood of the origin.

With the additional assumptions on $L(x, D)$ stated below, we shall give an alternative proof of this theorem. Throughout this paper we always assume that the order of $L(x, D)$ is $2 m(m \geqq 1)$. Moreover we substitute the conditions 1 and 2 by the following conditions $1^{\prime}$ and $2^{\prime}$ respectively:

Condition $1^{\prime} . \quad L_{0}\left(x^{\prime \prime}, y ; D\right)$ is strongly elliptic for $\left|x^{\prime \prime}\right|+|y|=1$ i.e. there exists a positive constant $\delta$ such that

$$
\operatorname{Re} L_{0}^{0}\left(x^{\prime \prime}, y ; \xi, \eta\right) \geqq \delta\left(|\xi|^{2}+|\eta|^{2}\right)^{2 m}
$$

for all $\left(x^{\prime \prime}, y\right)\left(\left|x^{\prime \prime}\right|+|y|=1\right), \xi \in \boldsymbol{R}^{k}$ and $\eta \in \boldsymbol{R}^{n}$. Here $L_{0}^{0}$ denotes the homogeneous part of $L_{0}$ of order $2 \mathrm{~m}$.

Condition $2{ }^{\prime}$. The differential equation

$$
\hat{L}_{0}\left(x^{\prime \prime}, y ; \xi, D_{y}\right) v(y)=0
$$

has no non-trivial solution in $L^{2}\left(\boldsymbol{R}_{y}^{n}\right)$ for all $x^{\prime \prime}$ and $\xi(|\xi|=1)$.

THEOREM 1.1'. Assume that $\rho_{0}>\sigma^{0}$. Under the conditions $1^{\prime}$ and $2^{\prime}$, the operator $L(x, D)$ is hypoelliptic in a neighborhood of the origin of $\boldsymbol{R}^{N}$.

Remark. Grušin showed in [3] that Condition 2 is equivalent to Condition 2' under Condition 1.

In order to show the hypoellipticity of the operator $L(x, D)$ it is essential to obtain the inequality of the type (2.13) as will be explained in $\S 2$. In $\S 3$, we shall get this inequality with the aid of the method suggested by that used in [13].

\section{\$2. The proof of Theorem $1.1^{\prime}$.}

In this section, according to $[5, \S 2]$, we shall describe in several steps how the proof of Theorem $1.1^{\prime}$ (or Theorem 1.1) is deduced to prove the inequality (2.13).

(A) Let $H_{1}$ and $H_{2}$ be two Hilbertian spaces and we denote by $\mathscr{L}\left(H_{1}, H_{2}\right)$ the set of all continuous linear mappings from $H_{1}$ into $H_{2}$. $\mathscr{L}\left(H_{1}, H_{2}\right)$ is a Banach space with the usual norm (denoted by $\|\cdot\|$ ). Let $p(x, \xi)$ be $\mathscr{L}\left(H_{1}, H_{2}\right)$-valued infinitely differentiable function defined in $\Omega \times \boldsymbol{R}_{\xi}^{k}$, where $\Omega$ is an open set in $\boldsymbol{R}_{x}^{k}$. We suppose that for every com- 
pact set $K \subset \Omega$ and for all multi-indices $\alpha, \beta$, the following inequality holds :

$$
\left\|p_{(\beta)}^{(\alpha)}(x, \xi)\right\| \leqq C_{\alpha, \beta}(1+|\xi|)^{m-\rho|\alpha|+\delta|\beta|} \quad x \in K, \xi \in R^{k},
$$

where $\rho$ and $\delta$ are non-negative numbers and

$$
p_{(\beta)}^{(\alpha)}(x, \xi)=\frac{\partial^{\alpha+\beta}}{\partial x^{\beta} \partial \xi^{\alpha}} p(x, \xi) .
$$

We denote by $S_{\rho, \delta}^{m}=S_{\rho, \delta}^{m}\left(\Omega \times R_{\xi}^{k} ; H_{1}, H_{2}\right)$ the set of all $p(x, \xi)$ satisfying the above conditions. We denote by $\mathscr{D}\left(\Omega ; H_{1}\right)$ the set of $H_{1}$-valued infinitely differentiable functions with compact support in an open set $\Omega \subset \boldsymbol{R}_{x}^{k}$. Then we define a pseudo-differential operator:

$$
p(x, D) u=(2 \pi)^{-k} \int_{R_{\xi}^{k}} p(x, \xi) \hat{u}(\xi) e^{i\langle x, \xi\rangle} d \xi,
$$

where $\hat{u}(\xi)$ is the Fourier transform of $u \in \mathscr{D}\left(\Omega ; H_{1}\right)$. For this pseudodifferential operator almost all theorems proved in [8] in the scaler case are true.

Now consider a function $\mu(\xi)$ infinitely differentiable except at $\xi=0$ such that $\operatorname{grad}_{\xi} \mu(\xi) \rightarrow 0$ as $\xi \rightarrow \infty$ and that the following inequality holds:

$$
C_{1}(1+|\xi|)^{s_{1}} \leqq \mu(\xi) \leqq C_{2}(1+|\xi|)^{s_{2}}, \quad \xi \in R^{k},
$$

where $0<\varepsilon_{1} \leqq \varepsilon_{2} \leqq 1$ and $C_{1}, C_{2}>0$.

THEOREM 2.1 (cf. [5, Theorem 1.1]; [6], [8]). Let $p(x, \xi)$ be a symbol in $S_{\rho, \delta}^{m}$ with $0 \leqq \delta<\varepsilon_{1} \leqq \varepsilon_{2}<\rho$. Suppose that there exist positive constants $d, A$ and a real number a such that for all $x \in \Omega, \alpha, \beta$ and $\xi,|\xi|>A$, the following inequalities are valid:

$$
\begin{gathered}
\left|p_{(\beta)}^{(\alpha)}(x, \xi) v\right|_{H_{2}} \leqq C_{\alpha, \beta} \mu(\xi)^{-|\alpha|+|\beta|}|\xi|^{-\alpha|\alpha+\beta|}|p(x, \xi) v|_{H_{2}} \quad v \in H_{1}, \\
|\xi|^{a}|v|_{H_{1}} \leqq C|p(x, \xi) v|_{H_{2}} \quad v \in H_{1} .
\end{gathered}
$$

Then the pseudo-differential operator $p(x, D)$ is hypoelliptic in $\Omega$.

In what follows we shall use the symbols $C, A, \mu, \delta, \ldots$ to denote constants, and suffix or prime will also be used if necessary.

(B) Let $\Omega^{\prime}$ be an open neighborhood of the origin in $\boldsymbol{R}_{x^{\prime}}^{k}$ and let $B_{\mu}$ $=\left\{y \in \boldsymbol{R}_{y}^{n} ;|y|<\mu\right\}$. For every $x^{\prime} \in \Omega^{\prime}$ and $\xi \in \boldsymbol{R}_{\xi}^{k}$ we have

$$
\hat{L}\left(x^{\prime}, y, \xi, D_{y}\right) \in \mathscr{L}\left(\stackrel{\circ}{H}^{2 m}\left(B_{\mu}\right), L^{2}\left(B_{\mu}\right)\right) \text {. }
$$


Hence if we put

$$
p\left(x^{\prime}, \xi\right)=\hat{L}\left(x^{\prime}, y ; \xi, D_{y}\right),
$$

then $p\left(x^{\prime}, \xi\right) \in S_{1,0}^{2 m}\left(\Omega^{\prime} \times \boldsymbol{R}_{\xi}^{k} ; \stackrel{\circ}{H}^{2 m}\left(B_{\mu}\right), L^{2}\left(B_{\mu}\right)\right)$.

If the hypoellipticity of the pseudo-differential operator $p\left(x^{\prime}, D_{x^{\prime}}\right)$ in $\Omega^{\prime}$ is proved, then we can derive the hypoellipticity of $L(x, D)$ in $\Omega \times B_{\mu}$ as follows:

LEMMA 2.1. Assume that Condition $1^{\prime}$ is satisfied and $p\left(x^{\prime}, D_{x^{\prime}}\right)$ is hypoelliptic in $\Omega^{\prime}$. Then the differential operator $L(x, D)=L\left(x^{\prime}, y, D_{x^{\prime}}, D_{y}\right)$ is hypoelliptic in a neighborhood of the origin of $\boldsymbol{R}_{x^{\prime}}^{k} \times \boldsymbol{R}_{y}^{n}=\boldsymbol{R}^{N}$.

Proof. By Condition $1^{\prime} L$ is elliptic in $\Omega^{\prime} \times B_{\mu}, y \neq 0$ (letting the sets $\Omega^{\prime}$ and $B_{\mu}$ shrink if necessary), therefore $L$ is hypoelliptic in $\Omega^{\prime} \times B_{\mu}$, $y \neq 0$. For any vector $\left(0, \cdots, 0, \eta_{1}, \cdots, \eta_{n}\right)=(0, \eta) \neq 0$, we can easily see the characteristic polynomial of $L_{0}$ at $y=0$ does not vanish by Condition $1^{\prime}$ :

$$
\sum_{\substack{|\alpha|=2 m \\ \alpha=\left(0, \alpha_{1}, \cdots, \alpha_{n}\right)}} a_{\alpha, 0}(0) \eta^{\alpha} \neq 0
$$

Thus $L$ is partially hypoelliptic with respect to the plane $y=0$, whence we have the conclusion of Lemma 2.1. (cf. [7, Chap. 4].)

(C) We introduce several notations. First we set

$$
\theta=\langle\sigma, \gamma\rangle+2 m-\langle\rho, \alpha\rangle .
$$

Then $\theta=0$ if and only if $(\alpha, \gamma) \in \mathfrak{M}_{0}$. (In the definition of $\mathfrak{M}:(1.1)$ and $\mathfrak{M}_{0}:(1.2)$, the number $m$ must be substituted by $2 m$.) We set

$$
\begin{gathered}
x^{\prime}=\left(x_{1}, \cdots, x_{k}\right), \quad y=\left(x_{k+1}, \cdots, x_{N}\right)=\left(y_{1}, \cdots, y_{n}\right) . \\
\left|x^{\prime}\right|_{\sigma^{\prime}}=\left|x_{1}\right|^{1 / \sigma_{1}}+\cdots+\left|x_{k}\right|^{1 / \sigma_{k}}, \\
|x|_{\sigma}=\left|x^{\prime}\right|_{\sigma^{\prime}}+\left|y_{1}\right|+\cdots+\left|y_{n}\right| \\
|\xi|_{\rho}=\left|\xi_{1}\right|^{1 / \rho_{1}}+\cdots+\left|\xi_{k}\right|^{1 / \rho_{k}} \\
h\left(x^{\prime \prime}, y ; \xi\right)=|x|_{\sigma}^{\rho_{1}-1}\left|\xi_{1}\right|+\cdots+|x|_{o}^{\rho_{k}-1}\left|\xi_{k}\right|
\end{gathered}
$$

and

$$
\rho_{0}=\min _{1 \leqq j \leqq k} \rho_{j}, \quad \rho^{0}=\max _{1 \leqq j \leqq k} \rho_{j}, \quad \sigma_{0}=\min _{1 \leqq j \leqq k} \sigma_{j}, \quad \sigma^{0}=\max _{1 \leqq j \leqq k} \sigma_{j} .
$$

Here $|x|_{\sigma}$ and $\left|x^{\prime}\right|_{\sigma}$ include only the terms corresponding to $\sigma_{j} \neq 0$.

LEMMA 2.2 ([5, Lemma 3.1]). Let $\alpha=\left(\alpha^{\prime}, \beta\right)=\left(\alpha_{1}, \cdots, \alpha_{k}, \beta_{1}, \cdots, \beta_{n}\right)$ and assume that 


$$
|\alpha|+\frac{\theta}{\rho_{0}} \leqq 2 m
$$

Then we have the following inequality:

$$
\left|x^{r} \xi^{\alpha^{\prime}}\right||\xi|_{\rho}^{\theta} \leqq C\left(|\xi|_{\rho}+h\left(x^{\prime \prime}, y ; \xi\right)\right)^{2 m-|\beta|} .
$$

LEMMA 2.3 ([5, Lemma 3.2]). Assume that

$$
|\alpha|+\frac{\theta}{\rho_{0}} \geqq 2 m \text {. }
$$

Then for arbitrary $\mu>0$ there exists a constant $C>0$ such that

$$
\begin{aligned}
\left|x^{r} \xi^{\alpha^{\prime}}\right||\xi|_{\rho}^{(2 m-|\alpha|) \rho_{0}} \leqq C\left(|\xi|_{\rho}+h\left(x^{\prime \prime}, y ; \xi\right)\right)^{2 m-|\beta|}, \quad|\xi| \geqq 1, \\
y \in B_{\mu}=\{y|| y \mid \leqq \mu\} .
\end{aligned}
$$

(D) In $\S 3$ we shall prove the following lemma.

LEMMA 2.4 (cf. [5, Lemma 3.5]). Assume that the conditions in Theorem 1.1' are satisfied. Then there exist positive constants $A, C$ and $\mu$ and a neighborhood $\Omega^{\prime}$ of the origin in $\boldsymbol{R}^{k}$ such that

$$
\begin{gathered}
\sum_{|\beta| \leqq 2 m}\left\|\left(|\xi|_{\rho}+h\left(x^{\prime \prime}, y, \xi\right)\right)^{2 m-|\beta|} D_{y}^{\beta} v(y)\right\|_{L^{2}\left(\boldsymbol{R}^{n}\right)}^{2} \\
\leqq C\left\|\hat{L}\left(x^{\prime}, y ; \xi, D_{y}\right) v(y)\right\|_{L^{2}\left(\boldsymbol{R}^{n}\right)}^{2}
\end{gathered}
$$

for all $x^{\prime} \in \Omega^{\prime}, v(y) \in \stackrel{\circ}{H}^{2 m}\left(B_{\mu}\right)$ and $\xi,|\xi|_{\rho} \geqq A$.

Now by virtue of the inequality (2.13) we can see that the operator $p\left(x^{\prime}, \xi^{\prime}\right)=\hat{L}\left(x^{\prime}, y ; \xi, D_{y}\right):(2.6)$ satisfies the conditions of Theorem 2.1. In fact, we shall estimate $p_{\left(\beta_{1}\right)}^{\left(\alpha_{1}\right)}\left(x^{\prime}, \xi\right) v(y)$, where $v(y) \in \stackrel{\circ}{H}^{2 m}\left(B_{\mu}\right)$ and $\alpha_{1}, \beta_{1}$ are multi-indices in $N^{k}$. Since $p\left(x^{\prime}, \xi\right)$ is expressed as a sum of terms $a_{\alpha \gamma}(x) \xi^{\alpha^{\prime}} D_{y}^{\beta}, p_{\left(\beta_{1}\right)}^{\left(\alpha_{1}\right)}$ is a sum of terms

$$
\left(\frac{\partial}{\partial x^{\prime}}\right)^{\beta_{1}}\left(a_{\alpha \gamma}(x) x^{r}\right)\left(\frac{\partial}{\partial \xi}\right)^{\alpha_{1}}\left(\xi^{\alpha^{\prime}}\right) D_{y}^{\beta} v(y)
$$

Considering $\langle\sigma, \gamma\rangle-\langle\rho, \alpha\rangle+2 m \geqq 0$, the number $\theta$ corresponding to the term (2.14) is not smaller than $\left\langle\rho^{\prime}, \alpha_{1}\right\rangle-\left\langle\sigma^{1}, \beta_{1}\right\rangle$, where $\rho^{\prime}=\left(\rho_{1}, \cdots, \rho_{k}\right)$ and $\sigma^{\prime}=\cdots, \sigma_{k}$ ). Then by Lemma 2.2 and Lemma $2.3 L^{2}$-norm of (2.14) is estimated by

$$
C|\xi|_{\rho}^{-\left\langle\rho^{\prime}, \alpha_{1}\right\rangle+\left\langle\sigma^{\prime}, \beta_{1}\right\rangle}\left\|\left(|\xi|_{\rho}+h\right)^{2 m-|\beta|} D_{y}^{\beta} v(y)\right\|_{L^{2}(\beta \mu)} \quad|\xi|_{\rho} \geqq A .
$$

Hence we have by (2.13) 


$$
\|(\mathbf{2 . 1 4})\|_{L^{2}\left(B_{\mu}\right)} \leqq C|\xi|_{\rho}^{-\rho_{0}\left|\alpha_{1}\right|+\alpha^{0}\left|\beta_{1}\right|}\left\|\hat{L}\left(x^{\prime}, y ; \xi, D_{y}\right) v(y)\right\|_{L^{2}\left(B_{\mu}\right)},
$$

$|\xi|_{\rho} \geqq A$.

From this we can easily see that $p\left(x^{\prime}, \xi\right)$ satisfies the condition of Theorem 2.1 by taking

$$
\begin{gathered}
\sigma^{0}<\varepsilon<\rho_{0}, \\
d=\min \left(\rho_{0}-\varepsilon, \varepsilon-\sigma_{0}\right)
\end{gathered}
$$

and

$$
\mu(\xi)=\left[\left(1+\xi_{1}^{2}\right)^{1 / \rho_{1}}+\cdots+\left(1+\xi_{k}^{2}\right)^{1 / \rho_{k}}\right]^{e} .
$$

Thus to complete the proof of Theorem $1.1^{\prime}$, it remains to prove Lemma 2.4.

\section{§3. Proof of Lemma 2.4: Main estimate.}

Lemma 2.4 will be proved as a consequence of the following lemma.

Lemma 3.1. Let $L_{0}\left(x^{\prime \prime}, y, D\right)$ be given as in Theorem 1.1'. Then there exists a positive constant $C$ such that

$$
\begin{gathered}
\sum_{|\beta| \leq 2 m}\left\|\left(|\xi|_{\rho}+h\left(x^{\prime \prime}, y ; \xi\right)\right)^{2 m-|\beta|} D_{y}^{\beta} v(y)\right\|_{L^{2}\left(R^{n}\right)}^{2} \\
\quad \leqq C\left\|\hat{L}_{0}\left(x^{\prime \prime}, y ; \xi, D_{y}\right) v(y)\right\|_{L^{2}\left(\boldsymbol{R}^{n}\right)}^{2}
\end{gathered}
$$

for all $v(y) \in H^{2 m}\left(\boldsymbol{R}^{n}\right) \cap \mathscr{E}^{\prime}\left(\boldsymbol{R}^{n}\right)$ and $\xi \in \boldsymbol{R}^{k}$.

Proof. In (3.1) substitute $\xi$ by $\lambda^{\rho^{\prime}} \xi, x$ by $\lambda^{-\sigma} x$ and $D_{y}$ by $\lambda D_{y}$ respectively $(\lambda>0)$. Then we see that quasi-homogeneous order of both sides and is $2 m$. Hence it is sufficient to show the inequality (3.1) for $|\xi|_{\rho}=$ 1. Let $t(r)$ be an infinitely differentiable function in $r \geqq 0$ such that

$$
t(r)= \begin{cases}0 & 0 \leqq r \leqq 1 \\ r & r \geqq 2\end{cases}
$$

Define

$$
h_{1}\left(x^{\prime \prime}, y ; \xi\right)=\left(\left|x^{\prime}\right|_{\sigma^{\prime}}+t(|y|)\right)^{\rho_{1-1}}\left|\xi_{1}\right|+\cdots+\left(\left|x^{\prime}\right|_{\sigma^{\prime}}+t(|y|)^{\rho_{k}-1}\left|\xi_{k}\right| .\right.
$$

Then $h_{1}$ has the same order as $h$ in $|y|$ for large $|y|$ and is constant when $x^{\prime \prime}, \xi$ are fixed and $y$ runs through the sphere in $\boldsymbol{R}_{y}^{n}$. Furtheremore for $j \geqq 1$, 


$$
\begin{array}{ll}
\left|\frac{\partial^{j} h_{1}}{\partial r^{j}}\right| h_{1}^{-j} & \text { is bounded }, \\
\left|\frac{\partial^{j} h_{1}}{\partial r^{j}}\right| h_{1}^{-j-1} \rightarrow 0 & \text { as }\left|x^{\prime \prime}\right|+|y| \rightarrow \infty .
\end{array}
$$

Substituting $h$ by $h_{1}$ in (3.1) we obtain the inequality equivalent to (3.1). Put

$$
g\left(x^{\prime \prime}, r ; \xi\right)=\int_{0}^{r}\left(1+h_{1}\left(x^{\prime \prime}, r ; \xi\right)\right) d r
$$

which has the same order as $r h_{1}$ as $r \rightarrow \infty$.

Now we shall use the coordinate transformation introduced in [5], [16]:

$$
z=\frac{y}{|y|} g\left(x^{\prime \prime},|y| ; \xi\right)
$$

Then we have

$$
\frac{\partial z_{j}}{\partial y_{i}}=\frac{g\left(x^{\prime \prime}, y ; \xi\right)}{|y|} \delta_{i j}+\left(y_{i} y_{j}-\frac{g\left(x^{\prime \prime},|y| ; \xi\right)}{|\hbar|^{3}}+\frac{1+h_{1}\left(x^{\prime \prime},|y| ; \xi\right)}{|y|^{2}}\right),
$$

and the Jacobian of the transformation is

$$
\frac{\partial\left(z_{1} \cdots z_{n}\right)}{\partial\left(y_{1}, \cdots, y_{n}\right)}=\frac{g\left(x^{\prime \prime},|y| ; \xi\right)^{n-1}}{|y|^{n-1}}\left(1+h_{1}\left(x^{\prime \prime},|y| ; \xi\right)\right)
$$

We now pass from the variables $y$ to the new variables $z$ in the equation

$$
\hat{L}_{0}\left(x^{\prime \prime}, y ; \xi, D_{y}\right) v(y)=f(y) \text {. }
$$

It will have the form:

$$
M\left(z, D_{z}\right) v_{1}(z)=f_{1}(z),
$$

where

$$
\left\{\begin{array}{l}
v_{1}=\left(1+h_{1}\right)^{m-n / 2} v \\
f_{1}=\left(1+h_{1}\right)^{-m-n / 2} f
\end{array}\right.
$$

In the following we shall show the equation (3.8) is uniformly elliptic in $\boldsymbol{R}_{z}^{n}$ and all the coefficients belong to $\mathscr{B}\left(\boldsymbol{R}_{z}^{n}\right)$. From Condition $1^{\prime}$ it follows that for the principal part of $L_{0}$ (denoted by $\left.L_{0}^{0}\right)$ :

$$
\operatorname{Re} L_{0}^{0}\left(x^{\prime \prime}, y ; \xi, \eta\right) \geqq \delta\left(|\eta|^{2 m}+h\left(x^{\prime \prime}, y ; \xi\right)^{2 m}\right) \text {. }
$$




\section{Considering}

$$
M\left(z, D_{z}\right) v_{1}=\left(1+h_{1}\right)^{-m-n / 2} L_{0}\left(1+h_{1}\right)^{-m+n / 2} v_{1}=f_{1}
$$

we have for the principal part of $M$ (denoted by $M^{0}$ ):

$$
\operatorname{Re} M^{0}(z, \zeta) \geqq \delta\left(\sum_{i=1}^{n}\left|\sum_{j=1}^{n}\left(1+h_{1}\right)^{-1} \frac{\partial z_{i}}{\partial y_{j}} \zeta_{j}\right|^{2}\right)^{m}
$$

Furthermore by (3.5) and (3.6) we have

$$
\begin{gathered}
\left|\frac{\partial z_{j}}{\partial y_{i}}\left(1+h_{1}\right)^{-1}\right| \leqq C, \quad z \in R_{z}^{n}, \\
C^{\prime-1} \leqq\left(1+h_{1}\right)^{-n} \frac{\partial\left(z_{1} \cdots z_{n}\right)}{\partial\left(y_{1}, \cdots, y_{n}\right)} \leqq C^{\prime}
\end{gathered}
$$

for some positive constants $C$ and $C^{\prime}$. Hence we have

$$
\operatorname{Re} M^{0}(z, \zeta) \geqq \delta^{\prime}|\zeta|^{2 m} \quad \zeta \in R_{\zeta}^{n}
$$

for some constant $\delta^{\prime}>0$. Hence $M\left(z, D_{z}\right)$ is uniformly strongly elliptic in $\boldsymbol{R}_{z}^{n}$. We remark that $M\left(z, D_{z}\right)$ depends on $\left(x^{\prime \prime}, \xi\right)$ which is viewed as a parameter.

By the above consideration all the coefficients of $2 m$-th order in $M\left(z, D_{z}\right)$ are bounded in $\boldsymbol{R}_{z}^{n}$. The first derivatives of them are given in the form:

$$
\begin{aligned}
(1+ & \left.h_{1}\right)^{-2 m-1} \frac{\partial h_{1}}{\partial z_{k}} a_{\alpha}(0) \sum C_{\alpha, i_{1} \cdots i_{2 m}, j_{1} \cdots j_{2 m}} \prod_{\nu=1}^{2 m}\left(\frac{\partial z_{j_{\nu}}}{\partial y_{i_{\nu}}}\right) \\
& +\left(1+h_{1}\right)^{-2 m} a_{\alpha}(0) \sum C_{\alpha, i_{1} \cdots i_{2 m}, j_{1} \cdots j_{2 m}} \prod_{\nu=1}^{2 m}\left(\frac{\partial z_{j_{\nu}}}{\partial y_{i_{\nu}}}\right) \frac{\partial}{\partial z_{k}}\left(\frac{\partial z_{j_{\nu}}}{\partial y_{i_{\nu}}}\right),
\end{aligned}
$$

where the summations are taken for $i_{1}, \cdots, i_{2 m}, j_{1}, \cdots, j_{2 m}$ which run through 1, ..,n. By using the following inequalities derived by (3.5) and (3.6):

$$
\begin{aligned}
\left(1+h_{1}\right)\left|\frac{\partial h_{1}}{\partial z_{k}}\right| & \leqq C\left(1+h_{1}\right)^{-2}\left|\frac{\partial h_{1}}{\partial r}\right|, \\
\left|\frac{\partial z_{j}}{\partial y_{j}}\right| & \leqq C^{\prime}\left(1+h_{1}\right),
\end{aligned}
$$

and by using (3.3) we can finally see that (3.13) is bounded in $\boldsymbol{R}_{z}^{n}$. The boundedness of the higher derivatives are shown as above. Similarly, we can show recurcively that all the coefficients of $2 m-j$-th order $(j=$ 
$1,2, \cdots, 2 m$ ) belong to $\mathscr{B}\left(\boldsymbol{R}_{z}^{n}\right)$ using (2.10), (3.3), (3.4), (3.5), (3.6) and (3.12). Hence the Gårding inequality for $M\left(z, D_{z}\right)$ can be derived; namely there exist two constants $C_{1}>0$ and $C_{2} \geqq 0$ such that

$$
\begin{aligned}
\operatorname{Re}\left(M\left(z, D_{z}\right) v_{1}(z), v_{1}(z)\right) \geqq & C_{1}\left\|v_{1}(z)\right\|_{H^{m}\left(\boldsymbol{R}_{z}^{n}\right)}^{2}-C_{2}\left\|v_{1}(z)\right\|_{L^{2}\left(\boldsymbol{R}_{z}^{n}\right)} \\
& v_{1} \in H^{m}\left(\boldsymbol{R}_{z}^{n}\right) \cap \mathscr{E}^{\prime}\left(\boldsymbol{R}_{z}^{n}\right) \text { (cf. [14], p. 240) . }
\end{aligned}
$$

Here the constants $C_{1}$ and $C_{2}$ are independent of $\left(x^{\prime \prime}, \xi\right),|\xi|_{\rho}=1$.

We can substitute $v_{1}$ in $(3.14)$ by $\left(1+h_{1}\right)^{m} v_{1}(z)$ :

$$
\begin{aligned}
& \operatorname{Re}\left(M\left(z, D_{z}\right)\left(1+h_{1}\right)^{m} v_{1}(z),\left(1+h_{1}\right)^{m} v_{1}(z)\right) \\
& \quad \geqq C_{1}\left\|\left(1+h_{1}\right)^{m} v_{1}(z)\right\|_{H^{m}\left(\boldsymbol{R}^{n}\right)}^{2}-C_{2}\left\|\left(1+h_{1}\right)^{m} v_{1}(z)\right\|_{L^{2}\left(\boldsymbol{R}^{n}\right)}^{2} .
\end{aligned}
$$

Using Leibniz' formula and integration by parts, the left-hand side of (3.15) is estimated from above by

$$
\begin{aligned}
C\left\{\left\|\left(1+h_{1}\right)^{m} M\left(z, D_{z}\right) v_{1}\right\| \cdot\left\|\left(1+h_{1}\right)^{m} v_{1}\right\|\right. \\
\left.+\left\|\left(1+h_{1}\right)^{m} v_{1}\right\|_{H^{m}} \cdot\left\|\left(1+h_{1}\right)^{m} v_{1}\right\|_{H}^{m-1}\right\} .
\end{aligned}
$$

By (3.15), (3.16) and by interpolating the inequality of the form

$$
\left\|\left(1+h_{1}\right)^{m} v_{1}\right\|_{H^{m-1}} \leqq \varepsilon\left\|\left(1+h_{1}\right)^{m} v_{1}\right\|_{H^{m}}+C(\varepsilon)\left\|\left(1+h_{1}\right)^{m} v_{1}\right\|_{L^{2}},
$$

we have

$$
\left\|\left(1+h_{1}\right)^{m} v_{1}\right\|_{H^{m}} \leqq C\left(\left\|\left(1+h_{1}\right)^{m} M\left(z, D_{z}\right) v_{1}\right\|_{L^{2}}+\left\|\left(1+h_{1}\right)^{m} v_{1}\right\|_{L^{2}}\right)
$$

for some constant $C>0$.

Now starting from (3.18) and (3.14), we can show, by induction in $|\beta|$, that the following inequality is valid:

$$
\begin{aligned}
& \sum_{|\beta| \leqq m}\left\|\left(1+h_{1}\right)^{m} D_{z}^{\beta} v_{1}\right\|_{H^{m}} \\
& \quad \leqq C^{\prime}\left\{\left\|\left(1+h_{1}\right)^{m} M\left(z, D_{z}\right) v_{1}\right\|_{L^{2}}+\left\|\left(1+h_{1}\right)^{m} v_{1}\right\|_{L^{2}}\right\} .
\end{aligned}
$$

Going over to the variables $y$ we have

$$
\begin{aligned}
\sum_{|\beta| \leqq 2 m} & \left\|\left(1+h_{1}\right)^{2 m-|\beta|} D_{y}^{\beta} v(y)\right\|_{L^{2}\left(\boldsymbol{R}^{n)}\right.} \\
\leqq & C^{\prime \prime}\left\{\left\|\hat{L}_{0}\left(x^{\prime \prime}, y ; \xi, D_{y}\right) v\right\|_{L^{2}}+\left\|\left(1+h_{1}\right)^{2 m} v\right\|_{L^{2}}\right\} \\
& v \in H^{2 m}\left(\boldsymbol{R}^{n}\right) \cap \varepsilon^{\prime}\left(\boldsymbol{R}^{n}\right),|\xi|_{\rho}=1 .
\end{aligned}
$$

The second term of the right hand side of (3.20) can be dropped out by Condition $2^{\prime}$, which completes the proof of Lemma 3.1 .

Proof of Lemma 2.4. The differential operator $\hat{L}\left(x^{\prime}, y ; D_{y}\right)$ is ex- 
pressed as a sum of the terms $a_{\alpha \gamma}(x) x^{r} \xi^{\alpha^{\prime}} D_{y}^{\beta},(\gamma, \alpha) \in \mathfrak{M}, \alpha=\left(\alpha^{\prime}, \beta\right)$. For $v \in \stackrel{\circ}{H}^{2 m}\left(B_{\mu}\right)$ we have

$$
\begin{aligned}
& \hat{L} v=\hat{L}_{0} v+\left(\hat{L}-\hat{L}_{0}\right) v \\
& =\hat{L}_{0} v+\sum_{\theta=0}\left(a_{\alpha \gamma}(x)-a_{\alpha \gamma}(0)\right) x^{\gamma} \xi^{\alpha^{\prime}} D_{y}^{\beta} v+\sum_{\theta>0} a_{\alpha \gamma}(x) x^{\gamma} \xi^{\alpha^{\prime}} D_{y}^{\beta} v .
\end{aligned}
$$

For any $\varepsilon>0$, if we take $\mu>0$ and if the diameter of $\Omega^{\prime} \subset R_{x}^{k}$ is sufficiently small, we have

$$
\begin{aligned}
& \sum_{\theta=0}\left\|\left(a_{\alpha \gamma}(x)-a_{\alpha \gamma}(0)\right) x^{\alpha} \xi^{\alpha^{\prime}} D_{y}^{\beta} v(y)\right\|_{L^{2}\left(B_{\mu}\right)} \\
& \quad \leqq \varepsilon \sum_{|\beta| \leqq 2 m}\left\|\left(|\xi|_{\rho}+h\left(x^{\prime \prime}, y ; \xi\right)\right)^{2 m-|\beta|} D_{y}^{\beta} v(y)\right\|_{L^{2}\left(B_{\mu}\right)}, \quad v \in \stackrel{\circ}{H}^{2 m}\left(B_{\mu}\right) .
\end{aligned}
$$

For the last sum in the right hand side of (3.21) we have by Lemma 2.2 and Lemma 2.3,

$$
\begin{aligned}
& \left\|a_{\alpha \gamma}(x) x^{\gamma} \xi^{\alpha^{\prime}} D_{y}^{\beta} v\right\|_{L^{2}\left(B_{\mu}\right)} \\
& \quad \leqq C_{\alpha, \gamma, \mu}|\xi|_{\rho}^{-\tau}\left\|\left(|\xi|_{\rho}+h\left(x^{\prime \prime}, y, \xi\right)\right)^{2 m-|\beta|} D_{y}^{\beta} v(y)\right\|_{L^{2}\left(B_{\mu}\right)}, \quad \tau>0 .
\end{aligned}
$$

If we take a sufficiently large number $A$, then we have

$$
\begin{aligned}
& \sum_{\theta=0}\left\|a_{\alpha \gamma}(x) x^{r} \xi^{\alpha^{\prime}} D_{y}^{\beta} v(y)\right\|_{L^{2}\left(B_{\mu}\right)} \\
& \quad \leqq \varepsilon \sum_{|\beta| \leqq 2 m}\left\|\left(|\xi|_{\rho}+h\left(x^{\prime \prime}, y, \xi\right)\right)^{2 m-|\beta|} D_{y}^{\beta} v(y)\right\|_{L^{2}\left(B_{\mu}\right)}, \quad|\xi|_{\rho} \geqq A .
\end{aligned}
$$

Thus by using Lemma 3.1, we are given desired inequality:

$$
\begin{aligned}
& \|\hat{L} v\|_{L^{2}\left(B_{\mu}\right)} \geqq\left\|\hat{L}_{0} v\right\|_{L^{2}\left(B_{\mu}\right)}-\left\|\left(\hat{L}-\hat{L}_{0}\right) v\right\|_{L^{2}\left(B_{\mu}\right)} \\
& \geqq(C-2 \varepsilon) \sum_{|\beta| \leq 2 m}\left\|\left(|\xi|_{\rho}+h\left(x^{\prime \prime}, y, \xi\right)\right)^{2 m-|\beta|} D_{y}^{\beta} v(y)\right\|_{L^{2}\left(B_{\mu}\right)}, \\
& v \in \stackrel{H}{ }^{2 m}\left(B_{\mu}\right),|\xi|_{\rho} \geqq A \text {. }
\end{aligned}
$$

Since $\varepsilon$ can be taken arbitrarily the inequality (2.13) is obtained.

\section{§ 4. Examples.}

The following examples have been given in [5].

1. $L=\frac{\partial^{2}}{\partial y^{2}}+y^{2} \frac{\partial^{2}}{\partial x^{2}}+\lambda \frac{\partial}{\partial x} \quad \operatorname{Re} \lambda \neq 0$ or $\operatorname{Re} \lambda=0$ and $|\lambda|<1$.

2. $L=\frac{\partial^{2}}{\partial y^{2}}+\left(x^{2}+y^{2}\right) \frac{\partial^{2}}{\partial x^{2}}$

These operators satisfy the conditions in Therem 1.1 and also $1.1^{\prime}$, hence they are hypoelliptic in $(x, y)$-plane. 


\section{Chapter II. Boundary value problems.}

\section{§ 5. Introduction.}

As an application of the method developed in Chapter I, we shall consider the regularity of solutions of boundary value problems for the degenerate elliptic equations being restricted to the second order.

Consider the case where $n=1$ and $m=2$. Set

$$
\boldsymbol{R}_{+}^{N}=\boldsymbol{R}^{k} \times \boldsymbol{R}_{+}^{1}=\left\{\left(x^{\prime}, y\right) \mid x^{\prime} \in \boldsymbol{R}^{k}, y>0\right\} .
$$

The letters $\rho, \sigma, \mathfrak{M}$ and $\mathfrak{M}_{0}$ will be used as in $\S 1$. Thus we shall consider a partial differential operator

$$
L(x, D)=\sum_{\substack{|\alpha| \leq 2 \\(\gamma, \alpha) \in \mathbb{M}}} a_{\alpha \gamma}(x) x^{r} D^{\alpha}, \quad a_{\alpha, r}(\dot{x}) \in C^{\infty}\left(\boldsymbol{R}_{+}^{N}\right)
$$

with one of the following boundary operators:

$$
\begin{gathered}
B_{1}\left(x^{\prime}, D_{x}\right) \equiv 1, \\
B_{2}\left(x^{\prime}, D_{x}\right)=D_{y}+b\left(x^{\prime}, D_{x^{\prime}}\right)+c(x) .
\end{gathered}
$$

Here $b\left(x^{\prime}, D_{x^{\prime}}\right)$ is a pseudo-differential operator defined in $\boldsymbol{R}^{k}$ with its symbol given by $\sum b_{r^{\prime}}\left(x^{\prime}\right) x^{\prime r^{\prime}} B_{r^{\prime}}(\xi)$, and $c\left(x^{\prime}\right)$ is a complex valued smooth function defined in $\boldsymbol{R}^{k}$. We shall, therefore, investigate the regularity of the solutions of the boundary value problem:

$$
\begin{gathered}
L\left(x, D_{x}\right) u(x)=f(x) \quad \text { in } R_{+}^{N}, \\
B_{j}\left(x^{\prime}, D_{x}\right) u\left(x^{\prime}, 0\right)=0 \quad \text { on } R_{x^{\prime}}^{k} \quad(j=1 \text { or } 2) .
\end{gathered}
$$

We freeze as in $\S 1$ the coefficients of the principal part of $L$ and $B_{2}$ and introduce the following notations:

$$
\begin{aligned}
\hat{L}_{0}\left(x^{\prime \prime}, y ; \xi, D_{y}\right) & =\sum_{\substack{|\alpha| \leq 2 \\
(\gamma, \alpha) \in \mathbb{M}_{0} \\
\alpha=\left(\alpha^{\prime}, \alpha_{N}\right)}} a_{\alpha, \gamma}(0) x^{r} \xi^{\alpha^{\prime}} D_{y}^{\alpha_{N}}, \\
\hat{B}_{2,0}\left(x^{\prime \prime}, \xi, D_{y}\right) & =D_{y}+\sum b_{\gamma^{\prime}}(0) x^{\prime \gamma^{\prime}} B_{r^{\prime}}(\xi) .
\end{aligned}
$$

Now we impose the following conditions.

Condition II. 1. $L_{0}\left(x^{\prime \prime}, y ; D\right)=\sum_{\substack{|\alpha| \leq 2 \\(\gamma, \alpha) \in \mathbb{M}_{0}}} a_{\alpha, r}(0) x^{r} D^{\alpha}$ is strongly elliptic for $\left|x^{\prime \prime}\right|+y=1$ with $y \geqq 0$.

Condition II. $2,(j=1$ or 2$)$. The homogeneous boundary value 
problem on the half line $\boldsymbol{R}_{+}^{1}$ :

$$
\hat{L}_{0}\left(x^{\prime \prime}, y ; D_{y}\right) v(y)=0,
$$

$$
\hat{B}_{j, 0}\left(x^{\prime \prime}, y ; \xi, D_{y}\right) v(0)=0
$$

has no non-trivial solution in $H^{2}\left(R_{+}^{1}\right)$ for all $x^{\prime \prime}$ and $\xi\left(|\xi|_{\rho}=1\right)$.

Condition II. 3. The symbol $b_{0}\left(x^{\prime \prime}, \xi\right)=\sum b_{r^{\prime}}(0) x^{\prime r^{\prime}} B_{r^{\prime}}(\xi)$ is quasihomogeneous of order one, that is,

$$
b_{0}\left(\lambda^{-\sigma^{\prime \prime}} x^{\prime \prime}, \lambda^{\rho^{\prime}} \xi\right)=\lambda b_{0}\left(x^{\prime \prime}, \xi\right) \quad \lambda>0 .
$$

Condition II. 4. $b\left(x^{\prime}, \xi\right)$ is real valued in $\boldsymbol{R}_{x^{\prime}}^{k}$.

THEOREM 5.1. Assume that $\rho_{0}>\sigma^{0}$ and that boundary value problem (5.3), (5.4) ${ }_{j}(j=1$ or 2$)$ satisfies either

case 1 . the conditions II. 1 and II. $2_{1}$

or

case 2. the conditions II. 1, II. 2 , II. 3 and II. 4 corresponding to $j=1$ or 2 .

If $\left.f \in C^{\infty} \overline{\left(\boldsymbol{R}_{+}^{N}\right.} \cap U\right)$ (where $U$ is a neighborhood of the origin in $\boldsymbol{R}^{N}$ ) and if $u \in H_{\mathrm{loc}}^{2} \overline{\left(\boldsymbol{R}_{+}^{N}\right)}$ with (5.3), (5.4), in $\boldsymbol{R}_{+}^{N} \cap U(j=1$ or 2$)$, then there exists a neighborhood $V$ of the origin such that $u \in C^{\infty}\left(\overline{\boldsymbol{R}_{+}^{N}} \cap V\right)$.

The proof of Theorem 5.1 can be reduced to that of the inequality (6.1) which will be proved in $\S 6$. The inequality (6.1) corresponds to (2.13) proved in Chapter I. By applying (6.1) and Theorem 2.1, we can immediately prove the regularity of $u$ at the boundary as in the case treated in Chapter I. In case the symbol $b\left(x^{\prime}, \xi\right)$ of the boundary opeyator $B_{2}$ is complex valued we need another method to obtain the estimate of the type (6.1). This method, which will be discussed in $\S 7$, can be applied for higher order operators. To illustrate our discussions we shall give in $\S 8$ examples of boundary systems satisfying the conditions in Theorem 5.1. Those conditions might be thought to be as close enough to a necessary condition.

\section{§ 6. Boundary estimates.}

As explained previously the proof of Theorem 5.1 can be reduced to the following lemma. 
LEMMA 6.1 (cf. Lemma 2.4). Assume that the conditions in Theorem 5.1 are satisfied. (i.e. $\rho_{0}>\sigma^{0}$, either the case 1: Conditions II. 1 and II. $2_{1}$ for the boundary system $\left\{L, B_{1}\right\}$, or the case 2: Conditions II. 1 , II. $2_{2}$, II. 3 and II. 4 for $\left.\left\{L, B_{2}\right\}\right)$. Then there exists positive constants $A, C$ and $\mu$ and a neighborhood $\Omega^{\prime}$ of the origin in $\boldsymbol{R}^{k}$ such that the following inequality holds:

$$
\begin{gathered}
\sum_{\beta=0}^{2}\left\|\left(|\xi|_{\rho}+h\left(x^{\prime \prime}, y ; \xi\right)\right)^{2-\beta} D_{y}^{\beta} v(y)\right\|_{L^{2}\left(I_{\mu}\right)}^{2} \\
\leqq C\left\|\hat{L}\left(x^{\prime}, y ; \xi, D_{y}\right) v(y)\right\|_{L^{2}\left(I_{\mu}\right)}^{2}
\end{gathered}
$$

for all $v \in \stackrel{\circ}{H}^{2}\left(I_{\mu}\right), I_{\mu}=[0, \mu)$, satisfiying

$$
\left.\hat{B}_{j}\left(x^{\prime} ; \xi, D_{y}\right) v(y)\right|_{y=0}=0 \quad(j=1 \text { or } 2),
$$

and for all $x^{\prime} \in \Omega^{\prime}$ and $\xi,|\xi|_{\rho} \geqq A$. Here

$$
h\left(x^{\prime \prime}, y ; \xi\right)=|x|_{\sigma}^{\rho_{1}-1}\left|\xi_{1}\right|+\cdots+|x|_{\sigma}^{\rho_{k}-1}\left|\xi_{k}\right| \quad \text { (see (2.8)) . }
$$

The proof will be obtained in several steps. Denote by $\mathscr{D}_{j}\left[\hat{L}_{0}\right](j=$ 1 or 2) the set of all $v=v(y) \in H^{2}\left(\boldsymbol{R}_{+}^{1}\right)$ such that supp $v$ is compact in $[0, \infty)$ and that

$$
\left.\hat{B}_{j, 0}\left(x^{\prime \prime}, \xi ; D_{y}\right) v(y)\right|_{y=0}=0 \quad(j=1 \text { or } 2 \text {, respectively }) .
$$

Lemma 6.1 will be proved as a consequence of the following two lemmas 6.2 and 6.3 .

LEMMA 6.2. Assume that the conditions in Theorem 5.1 are satisfied. Then there exist a constant $C$ and a neighborhood $\Omega^{\prime}$ of the origin in $\boldsymbol{R}^{k}$ such that

$$
\begin{gathered}
\sum_{\beta=0}^{2}\left\|\left(|\xi|_{\rho}+h\left(x^{\prime \prime}, y ; \xi\right)\right)^{2-\beta} D_{y}^{\beta} v(y)\right\|_{L^{2}\left(\boldsymbol{R}_{+}^{1}\right)}^{2} \\
\leqq C\left\|\hat{L}_{0}\left(x^{\prime \prime}, y ; \xi, D_{y}\right) v(y)\right\|_{L^{2}\left(\boldsymbol{R}_{+}^{1}\right)}^{2}
\end{gathered}
$$

for all $v \in \mathscr{D}_{j}\left[\hat{L}_{0}\right], x^{\prime} \in \Omega^{\prime}$ and $\xi \in \boldsymbol{R}^{k}$.

Proof. As in the proof of Lemma 3.1, the substitution: $\xi \rightarrow \lambda^{\rho} \xi$, $x \rightarrow \lambda^{-\sigma} x$ and $D_{y} \rightarrow \lambda D_{y}(\lambda>0)$ reduces the proof of (6.4) to the case $|\xi|_{\rho}=1$. We shall use the coordinate transformation as in $\S 3$ and use the same notations $h_{1}, g, \ldots$. In particular,

$$
z=g\left(x^{\prime \prime}, y ; \xi\right) \quad y \geqq 0,|\xi|=1 \text { : see }(3.4) \text {. }
$$


We bring the equation

$$
\hat{L}_{0}\left(x^{\prime \prime}, y ; \xi, D_{y}\right) v(y)=f(y) \quad y \geqq 0: \text { see }(3.7)
$$

into the form

$$
M\left(z, D_{z}\right) v_{1}=M\left(x^{\prime \prime}, \xi ; z, D_{z}\right) v_{1}(z)=f_{1}(z) \quad z \geqq 0,|\xi|_{\rho}=1: \text { see }(3.8)
$$

where $v_{1}(z)=\left(1+h_{1}\right)^{1-1 / 2} v$ and $f_{1}(z)=\left(1+h_{1}\right)^{-1-1 / 2} v$ as in (3.9). Considering a pair $\left(x^{\prime \prime}, \xi\right)\left(|\xi|_{\rho}=1\right)$ as a parameter, we observe that

$$
\begin{gathered}
M\left(z, D_{z}\right)=a_{1}\left(z, x^{\prime \prime}\right) D_{z}^{2}+a_{2}\left(z, x^{\prime \prime}, \xi\right) D_{z}+a_{3}\left(z, x^{\prime \prime}, \xi\right) \\
\operatorname{Re} a_{1}\left(z, x^{\prime \prime}, \xi\right) \geqq \delta>0: \quad \text { see }(3.10), \\
a_{j} \in B\left(R_{+}^{1}\right) \quad j=1,2,3 .
\end{gathered}
$$

The boundary condition $(5.6)_{j}(j=1$ or 2$)$ will change into

$$
\begin{gathered}
N_{1} v_{1}(0)=v_{1}(0)=0, \\
N_{2} v_{1}(0)=\left(D_{z}-\left(1+h_{1}\left(x^{\prime \prime}, 0 ; \xi\right)\right)^{-1} b_{0}\left(x^{\prime \prime}, \xi\right)\right) v_{1}(0)=0 \quad\left(|\xi|_{\rho}=1\right) .
\end{gathered}
$$

Since the boundary systems $\left\{a_{1} D_{z}^{2}+1,1\right\}$ and $\left\{a_{1} D_{z}^{2}+1, D_{z}\right\}$, considered in $\boldsymbol{R}_{+z}^{1}$, are stably variational (stablement variationnel) in the sense of Shimakura (cf. [15]), the Gårding inequality holds for $\left\{M\left(z, D_{z}\right), N_{j}\right\}$ $(j=1$ or 2$)$ :

$$
\begin{aligned}
\left\|v_{1}(z)\right\|_{H^{1}\left(R_{+}^{1}\right)}^{2} & \geqq C_{0} \operatorname{Re}\left(M\left(z, D_{z}\right) v_{1}(z), v_{1}(z)\right) \\
& \geqq C_{1}\left\|v_{1}(z)\right\|_{H^{1}\left(R_{+}^{1}\right)}^{2}-C_{2}\left\|v_{1}(z)\right\|_{L^{2}\left(R_{+}^{1}\right)}^{2} \quad v \in \mathscr{D}_{j}\left(\hat{L}_{0}\right) .
\end{aligned}
$$

Here the positive constants $C_{0}, C_{1}$ and $C_{2}$ can be chosen independently of $\left(x^{\prime \prime}, \xi\right)$ when $\left(x^{\prime \prime}, \xi\right)$ runs through a compact set. We shall prove the inequality (6.4) starting with (6.6). Let $v \in \mathscr{D}_{j}\left[\hat{L}_{0}\right](j=1$ or 2$)$, then we have $v_{1}(z) \in H^{2}\left(\boldsymbol{R}_{+}^{1}\right)$, supp $v_{1}$ is compact in $[0, \infty)$ and

$$
\begin{aligned}
\left.\left(1+h_{1}\right) v_{1}\right|_{z=1} & =0 \quad \text { if } v \in \mathscr{D}_{1}\left[\hat{L}_{0}\right]: \text { see }(6.5)_{1}, \\
\left.N_{2}\left(1+h_{1}\right) v_{1}\right|_{z=0} & =\left.\left(1+h_{1}\left(x^{\prime \prime}, 0 ; \xi\right)\right) N_{2}\left(x^{\prime \prime} \xi, D_{z}\right) v_{1}\right|_{z=0}=0 \\
& \text { if } v \in \mathscr{D}_{2}\left[\hat{L}_{0}\right]: \text { see }(6.5)_{2} .
\end{aligned}
$$

Thus we can substitute $v_{1}$ in $(6.6)$ by $\left(1+h_{1}\right) v_{1}(z)$ to prove

$$
\begin{aligned}
& \operatorname{Re}\left(M\left(z, D_{z}\right)\left(1+h_{1}\right) v_{1}(z),\left(1+h_{1}\right) v_{1}(z)\right) \\
& \quad \geqq C_{1}\left\|\left(1+h_{1}\right) v_{1}(z)\right\|_{H^{1}\left(R_{+}^{1}\right)}^{2}-C_{2}\left\|\left(1+h_{1}\right) v_{1}(z)\right\|_{L^{2}\left(R_{+}^{1}\right)}^{2} .
\end{aligned}
$$


Continuing the similar argument as in the proof of the inequality (3.18), we obtain

$$
\begin{aligned}
& \left\|\left(1+h_{1}\right) v_{1}(z)\right\|_{H^{1}\left(\boldsymbol{R}_{+}^{1}\right)} \\
& \quad \leqq C\left\{\left\|\left(1+h_{1}\right) M\left(z, D_{z}\right) v_{1}(z)\right\|_{L^{2}\left(\boldsymbol{R}_{+}^{1}\right)}+\left\|\left(1+h_{1}\right) v_{1}(z)\right\|_{L^{2}\left(\boldsymbol{R}_{+}^{1}\right)}\right\} \\
& \quad v \in \mathscr{D}_{j}\left[\hat{L}_{0}\right](j=1,2) .
\end{aligned}
$$

Since $a_{1} D_{z}^{2} v_{1}=f_{1}-\left(a_{2} D_{z} v_{1}+a_{3} v_{1}\right), \operatorname{Re} a_{1} \geqq \delta>0$, the $L^{2}$-norm of $\left(1+h_{1}\right) D_{z}^{2} v_{1}$ can be estimated by the right hand side of (6.8). Thus we have

$$
\begin{aligned}
& \sum_{\beta=0}^{2}\left\|\left(1+h_{1}\right) D_{z}^{\beta} v(z)\right\|_{L^{2}\left(R_{+}^{1}\right)} \\
& \qquad C^{\prime}\left\{\left\|\left(1+h_{1}\right) M\left(z, D_{z}\right) v_{1}(z)\right\|_{L^{2}\left(\boldsymbol{R}_{+}^{1}\right)}+\left\|\left(1+h_{1}\right) v_{1}(z)\right\|_{L^{2}\left(\boldsymbol{R}_{+}^{1}\right)}\right\} \\
& \quad v \in \mathscr{D}_{j}\left[\hat{L}_{0}\right](j=1 \text { or } 2),
\end{aligned}
$$

where the constant $C^{\prime}$ can be taken independently of $\left(x^{\prime \prime}, \xi\right)$ when it runs through a compact set. Now going over to the variable $y$, as in the proof of (3.18), and using Condition II. 2, we have the inequality (6.4).

LEMMA 6.3. Assume that $\rho_{0}>\sigma^{0}$ and that the boundary system $\left\{\hat{L}_{0}\left(x^{\prime \prime}, \xi ; D_{y}\right), \hat{B}_{0}\left(x^{\prime \prime}, \xi ; D_{y}\right)\right\}$ satisfies the Conditions II. 1, II. 2, II. 3 and II. 4. Consider the perturbed boundary system $\left\{\hat{L}_{0}\left(x^{\prime \prime}, \xi ; D_{y}\right), \hat{B}_{0}\left(x^{\prime \prime}, \xi ; D_{y}\right)+c\left(x^{\prime}\right)\right\}$ with parameter $\left(x^{\prime}, \xi\right)$ and with $c\left(x^{\prime}\right)$ given in $(5.2)_{2}$. Then for any $\mu>0$ and for any neighborhood $\Omega^{\prime}$ of the origin in $\boldsymbol{R}^{k}$, there exist positive constants $A$ and $C$ such that

$$
\begin{gathered}
\sum_{\beta=0}^{2} \|\left(|\xi|_{\rho}+h\left(x^{\prime \prime}, y ; \xi\right)^{2-\beta} D_{y}^{\beta} v(y) \|_{L^{2}\left(I_{\mu}\right)}^{2}\right. \\
\leqq C\left\|\hat{L}_{0}\left(x^{\prime \prime}, y ; \xi, D_{y}\right) v(y)\right\|_{L^{2}\left(I_{\mu}\right)}^{2}
\end{gathered}
$$

for all $v \in H^{2}\left(I_{\mu}\right), I_{\mu}=[0, \mu)$, satisfying

$$
\left[\hat{B}_{2,0}\left(x^{\prime \prime}, \xi, D_{y}\right)+c\left(x^{\prime}\right)\right] v(0)=\left[D_{y}+b_{0}\left(x^{\prime \prime}, \xi\right)+c\left(x^{\prime}\right)\right] v(0)=0
$$

and for all $x^{\prime} \in \Omega^{\prime}$ and $\xi,|\xi|_{\rho} \geqq A$.

Proof. Take $v \in \stackrel{\circ}{H}^{2}\left(I_{\mu}\right)$ satisfying the boundary condition (6.11). Put

$$
u(y)=v(y) \exp \left[i c\left(x^{\prime}\right) y\right] \text {. }
$$

Then we have $u(y) \in \stackrel{\circ}{ }^{2}\left(I_{\mu}\right)$ and

$$
\left.\hat{B}_{2,0}\left(x^{\prime \prime}, \xi, D_{y}\right) u(y)\right|_{y=0}=0 \text {. }
$$


By Lemma 6.2, we have

$$
\sum_{\beta=0}^{2}\left\|\left(|\xi|_{\rho}+h\right)^{2-\beta} D_{y}^{\beta} u\right\|_{L^{2}\left(I_{\mu}\right)} \leqq C^{\prime}\left\|\hat{L}_{0}\left(x^{\prime \prime}, y ; \xi, D_{y}\right) u\right\|_{L^{2}\left(I_{\mu}\right)}, \quad|\xi|_{\rho} \geqq A^{\prime}
$$

for some positive constants $C^{\prime}$ and $A^{\prime}$ independent of such $u$. From (6.12) we easily obtain the following inequality:

$$
\begin{aligned}
& \sum_{\beta=0}^{2}\left\|\left(|\xi|_{\rho}+h\right)^{2-\beta} D_{y}^{\beta} v\right\|_{L^{2}\left(I_{\mu}\right)} \\
& \quad \leqq C_{1}\left\|\hat{L}_{0}\left(x^{\prime \prime}, y ; \xi, D_{y}\right) v\right\|_{L^{2}\left(I_{\mu}\right)}+C_{2}\left\|\left(|\xi|_{\rho}+h\right) v\right\|_{L^{2}\left(I_{\mu}\right)},
\end{aligned}
$$$$
|\xi|_{\rho} \geqq A^{\prime}
$$

for some constants $C_{1}$ and $C_{2}$ which depend only on $\Omega^{\prime}\left(c\left(x^{\prime}\right)\right)$. Since $h \rightarrow \infty$ as $|\xi|_{\rho} \rightarrow \infty$, there exist positive constants $C$ and $A$ such that

$$
\sum_{\beta=0}^{2}\left\|\left(|\xi|_{\rho}+h\right)^{2-\beta} D_{y}^{\beta} v\right\|_{L^{2}\left(I_{\mu}\right)} \leqq C\left\|\hat{L_{0}} v\right\|_{L^{2}}, \quad|\xi|_{\rho} \geqq A,
$$

which proves the inequality (6.10).

Q.E.D.

Proof of Lemma 6.1. A) First we consider the boundary system $\left\{L, B_{1}\right\}=\{L, 1\}$ under Conditions II. 1 and II. $2_{1}$. By Lemma 6.2, we have the following inequality for any $\mu>0$ :

$$
\begin{aligned}
\sum_{\beta=0}^{2}\left\|(1+h)^{2-\beta} D_{y}^{\beta} v\right\|_{L^{2}\left(I_{\mu}\right)} \leqq C\left\|\hat{L}_{0}\left(x^{\prime \prime}, y ; \xi, D_{y}\right) v(y)\right\|_{L^{2}\left(I_{\mu}\right)}^{2} & \\
v & \in \stackrel{\circ}{H}^{2}\left(I_{\mu}\right), v(0)=0, I_{\mu}=[0, \mu) .
\end{aligned}
$$

For $v \in \stackrel{\circ}{H}^{2}\left(I_{\mu}\right)$, with $v(0)=0$, we have

$$
\begin{aligned}
L\left(x^{\prime}, y ; \xi, D_{y}\right) v= & \hat{L}_{0} v+\left(L-\hat{L}_{0}\right) v \\
= & \hat{L}_{0} v+\sum_{\theta=0}\left(a_{\alpha \gamma}(x)-a_{\alpha \gamma}(0)\right) x^{r} \xi^{\alpha^{\prime}} D_{y}^{\beta} v \\
& +\sum_{\theta>0} a_{\alpha \gamma}(x) x^{r} \xi^{\alpha^{\prime}} D_{y}^{\beta} v .
\end{aligned}
$$

Thus in a similar manner to the proof of Lemma 2.4 we have the inequality (6.1) for the boundary system $\{L, 1\}$.

B) We consider boundry system $\left\{L, B_{2}\right\}$ under Conditions II. 1, II. $2_{2}$, II. 3 and II. 4 . Let $\mu$ be a positive number determined later and let

$$
v \in \stackrel{\circ}{H}^{2}\left(I_{\mu}\right),\left.\quad \hat{B}\left(x^{\prime}, \xi, D_{y}\right) v\right|_{y=0}=0 .
$$

If we put

$$
u(y)=v(y) \exp \left[i\left(b\left(x^{\prime}, \xi\right)-b_{0}\left(x^{\prime \prime}, \xi\right)\right) y\right]
$$


Then we have $u(y) \in \dot{H}^{2}\left(I_{\mu}\right)$ and

$$
\left.\left[\hat{B}_{0}\left(x^{\prime \prime}, \xi, D_{y}\right)+c\left(x^{\prime}\right)\right] u(y)\right|_{y=0}=0 .
$$

We can apply Lemma 6.3 to $u$ to obtain

$$
\sum_{\beta=0}^{2}\left\|\left(|\xi|_{\rho}+h\right)^{2-\beta} D_{y}^{\beta} u\right\|_{L^{2}\left(I_{\mu}\right)} \leqq C\left\|\hat{L}_{0} u\right\|_{L^{2}\left(I_{\mu}\right)} \quad x^{\prime} \in \Omega^{\prime},|\xi|_{\rho} \geqq A
$$

for some constants $C$ and $A$. Since $\hat{L}_{0}=\hat{L}-\left(\hat{L}-\hat{L}_{0}\right)$, we have

$$
\left\|\hat{L}_{0} u\right\|_{L^{2}\left(I_{\mu}\right)} \leqq\|\hat{L} u\|_{L^{2}\left(I_{\mu}\right)}+\left\|\left(\hat{L}-\hat{L}_{0}\right) u\right\|_{L^{2}\left(I_{\mu}\right)} \text {. }
$$

The first term in the right hand side of (6.17) is estimated by

$$
\begin{gathered}
\text { const }\left(\left\|e^{-i d\left(x^{\prime}, \xi\right) y} \hat{L} v\right\|_{L^{2}\left(I_{\mu}\right)}+\left\|d\left(x^{\prime}, \xi\right)^{2} v\right\|_{L^{2}\left(I_{\mu}\right)}+\left\|d\left(x^{\prime}, \xi\right) D_{y} v\right\|_{L^{2}\left(I_{\mu}\right)}\right), \\
d\left(x^{\prime}, \xi\right)=b\left(x^{\prime}, \xi\right)-b_{0}\left(x^{\prime \prime}, \xi\right) .
\end{gathered}
$$

By Condition II. $4, d\left(x^{\prime}, \xi\right)=b\left(x^{\prime}, \xi\right)-b_{0}\left(x^{\prime \prime}, \xi\right)$ is real valued. For any $\varepsilon>0$, if we choose the diameter of $\Omega^{\prime}$ sufficiently small and apply the inequality (2.10), we have

$$
\begin{aligned}
& \left\|d\left(x^{\prime}, \xi\right)^{2} v\right\|_{L^{2}\left(I_{\mu}\right)}+\left\|d\left(x^{\prime}, \xi\right) D_{y} v\right\|_{L^{2}\left(I_{\mu}\right)} \\
& \quad \leqq \varepsilon \sum_{\beta=0}^{2}\left\|\left(|\xi|_{\rho}+h\left(x^{\prime \prime}, y ; \xi\right)\right)^{2-\beta} D_{y}^{\beta} v\right\|_{L^{2}\left(I_{\mu}\right)} .
\end{aligned}
$$

To the second term in the right hand side of (6.17), we can apply the the similar manner to the proof of Lemma 2.4. Consequently we can choose $\mu$ and $\Omega^{\prime}$ sufficiently small and $A$ sufficiently large so that

$$
\begin{aligned}
&\left\|\left(\hat{L}-\hat{L_{0}}\right) u\right\|_{L^{2}\left(I_{\mu}\right)} \leqq \varepsilon \sum_{\beta=0}^{2}\left\|\left(|\xi|_{\rho}+h\right)^{2-\beta} D_{y}^{\beta} v\right\|_{L^{2}\left(I_{\mu}\right)}, \\
& \quad x^{\prime} \in \Omega^{\prime},|\xi|_{\rho} \geqq A .
\end{aligned}
$$

Similarly we have

$$
\sum_{\beta=0}^{2}\left\|\left(|\xi|_{\rho}+h\right)^{2-\beta} D_{y}^{\beta} u\right\|_{L^{2}\left(I_{\mu}\right)} \geqq(1-\varepsilon) \sum_{\beta=0}^{2}\left\|\left(|\xi|_{\rho}+h\right)^{2-\beta} D_{y}^{\beta} v\right\|_{L^{2}\left(I_{\mu}\right)} .
$$

Summing up the results $(6.16) \sim(6.21)$ we have the inequality of the form

$$
(1-2 \varepsilon) \sum_{\beta=0}^{2}\left\|\left(|\xi|_{\rho}+h\right)^{2-\beta} D_{y}^{\beta} v\right\|_{L^{2}\left(I_{\mu}\right)} \leqq C\|\hat{L} v\|_{L^{2}\left(I_{\mu}\right)},
$$

where $\varepsilon>0$ can be taken arbitrarily small if we choose $\mu, A>0$ and $\Omega^{\prime}$ adequately. This completes the proof of Lemma 6.1. 


\section{§ 7. Alternative method.}

Let $L(x, D)=\sum_{\substack{|\alpha| \leq 2 \\(\gamma, \alpha) \in \mathfrak{N}}} a_{\alpha \gamma}(x) x^{\tau} D^{\alpha}$ and let $B_{2}\left(x^{\prime}, D_{x}\right)=D_{y}+b\left(x^{\prime}, D_{y^{\prime}}\right)+$ $c\left(x^{\prime}\right)$. We assume the conditions II. 1, II. $2_{1}$, II. $2_{2}$ and II. 3 . In this section the symbol $b\left(x^{\prime}, \xi\right)$ can be complex-valued, since Conditions II. $2_{1}$ and II. $2_{2}$ are assumed simultaneously. We shall say that the boundary system $\left\{L(x, D), B_{2}\left(x^{\prime} D\right)\right\}$ is hypoelliptic at the boundary if the conclusion of Theorem 5.1 holds.

LEMMA 7.1. Under the conditions II. 1, II. $2_{1}$, II. $2_{2}$ and II. 3 , we have the same conclusion as in Lemma 6.1, that is, we have the inequality of the type (6.1).

As a consequences of Lemma 7.1 we have the following.

THEOREM 7.2. Assume that $\rho_{0}>\sigma^{0}$. Under the same conditions as in Lemma 7.1 the boundary system $\left\{L(x, D), B_{2}\left(x^{\prime}, D\right)\right\}$ is hypoelliptic at the boundary.

We shall first give the outline of the proof of Lemma 7.1 in several steps.

A) By the inequality (6.6) and the condition II: $2_{2}$ we have the following inequality for some positive constants $C$ and $A$ :

$$
\begin{array}{r}
\operatorname{Re}\left(\hat{L}_{0}\left(x^{\prime \prime}, y ; \xi, D_{y}\right) v(y), v(y)\right) \geqq C \sum_{\beta=0}^{1}\left\|\left(|\xi|_{\rho}+h\right)^{1-\beta} D_{y}^{\beta} v\right\|_{L^{2}(0, \infty)}^{2}, \\
|\xi|_{\rho} \geqq A, v \in \mathscr{D}_{2}\left[\hat{L}_{0}\right] .
\end{array}
$$

B) From the inequality (7.1) we derive the following inequality for some positive constants $\mathrm{C}, A$ and $\mu$ :

$$
\operatorname{Re}\left(\hat{L}\left(x^{\prime}, y ; \xi, D_{y}\right) v(y), v(y)\right)_{L^{2}(0, \mu)} \geqq C \sum_{\beta=0}^{1}\left\|\left(|\xi|_{\rho}+h\right)^{1-\beta} D_{y}^{\beta} v(y)\right\|_{L^{2}(0, \mu)}^{2}
$$

for $|\xi|_{\rho} \geqq A,\left|x^{\prime}\right| \leqq \mu$ and for $v \in \mathscr{D}_{2, \mu}[\hat{L}]$ which means that $v \in H^{2}(0, \mu)$ with $\operatorname{supp} v \subset[0, \mu)$ and satisfies

$$
\left[D_{y}+b\left(x^{\prime}, \xi\right)+c\left(x^{\prime}\right)\right] v(0)=0 .
$$

The inequality (7.2) might be viewed as an "coercive inequality" for the boundary system $\left\{L(x, D), B_{2}\left(x^{\prime}, D\right)\right\}$.

C) If $v \in \mathscr{D}_{2, u}[\hat{L}]$, then we obviously have $\left(|\xi|_{\rho}+h\left(x^{\prime \prime}, 0 ; \xi\right)\right) v(y) \in \mathscr{D}_{2, \mu}[\hat{L}]$. Substiting $v$ in $(7.2)$ with $\left(|\xi|_{\rho}+h\left(x^{\prime \prime}, 0 ; \xi\right)\right) v(y)$, we have 


$$
\sum_{\beta=0}^{1}\left\|\left(|\xi|_{\rho}+h\left(x^{\prime \prime}, y ; \xi\right)\right)^{1-\beta}\left(|\xi|_{\rho}+h\left(x^{\prime \prime}, 0 ; \xi\right)\right) D_{y}^{\beta} v(y)\right\|_{L^{2}(0, \mu)} \leqq C\|\hat{L} v\|_{L^{2}(0, \mu)}
$$

for $|\xi|_{\rho} \geqq A$ and $v \in \mathscr{D}_{2, \mu}[\hat{L}]$.

D) We have the following trace formula:

$$
\left(|\xi|_{\rho}+h\left(x^{\prime \prime}, 0 ; \xi\right)\right)^{1 / 2}|v(0)| \leqq C \sum_{\beta=0}^{1} \|\left(|\xi|_{\rho}+h\left(x^{\prime \prime}, y ; \xi\right)^{1-\beta} D_{y}^{\beta} v(y) \|_{L^{2}(0, \infty)}\right.
$$

for $v \in H^{1}(0, \infty)$ with compact support.

E) By the steps C) and D) the following inequality for some positive constants $C, A$ and $\mu$ is obtained:

$$
\begin{aligned}
\left(|\xi|_{\rho}+h\left(x^{\prime \prime}, 0 ; \xi\right)\right)^{3 / 2}|v(0)| \leqq C\|\hat{L} v\|_{L^{2}(0, \mu)}, & |\xi|_{\rho} \geqq A, v \in \mathscr{D}_{2, \mu}[\hat{L}] .
\end{aligned}
$$

F) For any $v \in \mathscr{D}_{2, \mu}[\hat{L}]$ there exists a function $w \in H^{2}(0, \mu)$ such that such that $w(0)=v(0)$, supp $w \subset[0, \mu)$ and

$$
\begin{aligned}
& \sum_{\beta=0}^{2}\left\|\left(|\xi|_{\rho}+h\left(x^{\prime \prime}, y ; \xi\right)\right)^{2-\beta} D_{y}^{\beta} w\right\|_{L^{2}(0, \mu)} \\
& \quad \leqq C\left(|\xi|_{\rho}+h\left(x^{\prime \prime}, 0 ; \xi\right)\right)^{3 / 2}|v(0)|, \quad\left|x^{\prime \prime}\right| \leqq \mu,
\end{aligned}
$$

where the constant $C$ is independent of $v \in \mathscr{D}_{2, \mu}[\hat{L}]$.

G) By (7.6). and (7.7) we have for another constant $C$

$$
\begin{aligned}
\sum_{\beta=0}^{2}\left\|\left(|\xi|_{\rho}+h\left(x^{\prime \prime}, y ; \xi\right)\right)^{2-\beta} D_{y}^{\beta} w\right\|_{L^{2}(0, \mu)} \leqq C\|\hat{L} v\|_{L^{2}(0, \mu)}, & |\xi|_{\rho} \geqq A, v \in \mathscr{D}_{2, \mu}[\hat{L}] .
\end{aligned}
$$

H) For $v \in \mathscr{D}_{2, \mu}[\hat{L}]$, we take $w$ defined in the step $\left.\mathrm{F}\right)$. Then $u=$ $v-w$ satisfies the Dirichlet condition: $u(0)=0$. By Condition II. $2_{1}$ and by the conclusion of Lemma 6.1 for the boundary system $\{L, 1\}$, we have the following inequality for some positive constants $C, A$ and $\mu$ :

$$
\|u\|_{2, \rho} \equiv \sum_{\beta=0}^{2}\left\|\left(|\xi|_{\rho}+h\left(x^{\prime \prime}, y \xi ; \xi\right)\right)^{2-\beta} D_{y}^{\beta} u(y)\right\|_{L^{2(0, \mu)}} \leqq C\|\hat{L} u\|_{L^{2}(0, \mu)},
$$

$|\xi|_{\rho} \geqq A$.

From this we have

$$
\|v\|_{2, \rho}-\|w\|_{2, \rho} \leqq C\left(\|\hat{L} v\|_{L^{2}(0, \mu)}+\|\hat{L} w\|_{L^{2}(0, \mu)}\right)
$$

and

$$
\|v\|_{2, \rho} \leqq C^{\prime}\left(\|\hat{L} v\|_{L^{2}(0, \mu)}+\|w\|_{2, \rho}\right)
$$

By (7.8) we have 


$$
\begin{aligned}
\sum_{\beta=0}^{2}\left\|\left(|\xi|_{\rho}+h\left(x^{\prime \prime}, y ; \xi\right)\right)^{2-\beta} D_{y}^{\beta} v(y)\right\|_{L^{2}(0, \mu)} \leqq C^{\prime \prime} & \|\hat{L} v\|_{L^{2}(0, \mu)}, \\
& |\xi|_{\rho} \geqq A, v \in \mathscr{D}_{2, \mu}[\hat{L}] .
\end{aligned}
$$

This proves Lemma 7.1.

Next we shall prove the inequalities left unproved in the above steps.

Proof of (7.2). Set $\mathscr{D}_{2, \mu}\left[\hat{L}_{0}\right]=\left\{v \mid v \in \mathscr{D}_{2}\left[\hat{L}_{0}\right]\right.$, supp $\left.v \subset[0, \mu)\right\}$ and write

$$
\begin{aligned}
\hat{L_{0}}\left(x^{\prime \prime}, \xi, D_{y}\right) & =a_{0}(0) D_{y}^{2}+a_{1,0}\left(x^{\prime \prime}, \xi\right) D_{y}+a_{2,0}\left(x^{\prime \prime}, \xi\right), \\
\hat{L}\left(x, \xi, D_{y}\right) & =a_{0}(x) D_{y}^{2}+a_{1}(x, \xi) D_{y}+a_{2}(x, \xi) .
\end{aligned}
$$

For $v \in \mathscr{D}_{2, \mu}\left[\hat{L}_{0}\right](\mu>0$ being determined later), we have

$$
\begin{aligned}
\operatorname{Re}\left(\hat{L}\left(x^{\prime}, y ; \xi, D_{y}\right) v(y), v(y)\right) \\
=\operatorname{Re}\left(\left(\hat{L}-\hat{L}_{0}\right) v, v\right)+\operatorname{Re}\left(\hat{L}_{0} v, v\right) \\
=\operatorname{Re}\left\{\left(\left(a_{0}\left(x^{\prime}, y\right)-a_{0}(0)\right) v^{\prime}(y), v^{\prime}(y)\right)\right. \\
\quad+\left(\left(i(\partial / \partial y) a_{0}\left(x^{\prime}, y\right)+a_{1}\left(x^{\prime}, y ; \xi\right)-a_{1,0}\left(x^{\prime \prime}, y ; \xi\right)\right) D_{y} v, v\right) \\
\left.\quad+\left(\left(a_{2}\left(x^{\prime}, y ; \xi\right)-a_{2}\left(x^{\prime \prime}, y ; \xi\right)\right) v(y), v(y)\right)\right\} \\
\quad+\operatorname{Re}\left\{\left(a_{0}(0) v^{\prime}(y), v^{\prime}(y)\right)+\left(a_{1,0}\left(x^{\prime \prime}, y ; \xi\right) D_{y} v, v\right)+\left(a_{2,0}\left(x^{\prime \prime}, y ; \xi\right) v, v\right)\right\} \\
\quad-\operatorname{Re} i b_{0}\left(x^{\prime \prime}, \xi\right) a_{0}(0)|v(0)|^{2}-\operatorname{Re} i b_{0}\left(x^{\prime \prime}, \xi\right)\left(a_{0}\left(x^{\prime}, 0\right)-a_{0}(0)\right)|v(0)|^{2}
\end{aligned}
$$

where we denote the inner product in $L^{2}(0, \mu)$ by $(\cdot, \cdot)$ and the norm in $L^{2}(0, \mu)$ by $\|\cdot\|$. We denote the last expression by $L[v, v]$. Taking $\mu>0$ sufficiently small and using the inequalities (7.1), (7.5), (2.10) and (2.12), we have for some positive constants $C$ and $A$

$$
L[v, v] \geqq C \sum_{\beta=0}^{1}\left\|\left(|\xi|_{\rho}+h\left(x^{\prime \prime} y \xi\right)\right)^{1-\beta} D_{y}^{\beta} v(y)\right\|_{L^{2}(0, \mu)}^{2}
$$

for all $v \in \mathscr{D}_{2, \mu}\left[\hat{L_{0}}\right]$ and $\left|x^{\prime}\right| \leqq \mu$. This inequality can be extended to all $v \in H^{1}(0, \mu)$ with supp $v \subset[0, \mu]$. On the other hand, (7.11) is valid for all $u \in \mathscr{D}_{2, \mu}[\hat{L}]$ since $\mathscr{D}_{2, \mu}[\hat{L}] \subset H^{1}(0, \mu)$ and $\operatorname{supp} u \subset[0, \mu)$. Now for $u \in \mathscr{D}_{2, \mu}[\hat{L}]$, we have

$$
L[u, u]=\operatorname{Re}(\hat{L} u, u)-\operatorname{Re} i\left(a_{0}\left(x^{\prime}, 0\right)\left(b_{0}\left(x^{\prime \prime}, \xi\right)-b\left(x^{\prime}, \xi\right)-c\left(x^{\prime}\right)\right)|u(0)|^{2} .\right.
$$

Again taking $\mu>0$ sufficiently small and by (7.5) we have the inequality (7.2).

Proof of F). It is sufficient to prove the case where $|\xi|_{p}=1$. Take a function $\psi \in C_{0}^{\infty}[0, \mu)$ such that $\psi(y) \equiv 10 \leqq y \leqq \mu / 2$. We make use of a transformation $y \rightarrow t$ given by 


$$
t=\int_{0}^{y}\left(1+h_{1}\left(x^{\prime \prime}, s ; \xi\right)\right) d s \quad y \geqq 0 .
$$

For $v \in H^{2}[0, \infty)$ set

$$
w(y)=\psi(t) v(0) .
$$

Then we have $w(0)=v(0)$ and

$$
\begin{aligned}
\sum_{\beta=0}^{2} \| & \left(1+h_{1}\left(x^{\prime \prime}, y ; \xi\right)\right)^{2-\beta} D_{y}^{\beta} w(y) \|^{2} \\
& \leqq C|v(0)|^{2} \sum_{\beta=0}^{2}\left\|\left(1+h_{1}\left(x^{\prime \prime}, y ; \xi\right)\right)^{3 / 2} D_{t}^{\beta} \psi(t)\right\|^{2} \\
& \leqq C^{\prime}|v(0)|^{2}\left(1+h_{1}\left(x^{\prime \prime}, 0 ; \xi\right)\right)^{3} \sum_{\beta=0}^{2} \| D_{t}^{\beta} \psi(t)_{L_{t}^{2}(0, \mu)}^{2} \\
& \leqq C^{\prime \prime}\left(1+h_{1}\left(x^{\prime \prime}, 0 ; \xi\right)\right)^{3}|v(0)|^{2}, \quad\left|x^{\prime \prime}\right| \leqq \mu
\end{aligned}
$$

From these the inequality (7.7) can be derived.

\section{§ 8. Examples.}

EXAMPLE 1. Consider the boundary value problem:

$$
\begin{gathered}
L v=\left(D_{y}^{2}+\left(x^{2 k}+y^{\ell}\right) D_{x}^{2}\right) u(x, y) \quad y>0, \quad-\infty<x<\infty, \\
\quad(k, \ell=1,2, \ldots), \\
\left.B_{2} u\right|_{y=0}=\left(D_{y}+b(x) x^{k} D_{x}+\left.c(x) u\right|_{y=0}=0,\right.
\end{gathered}
$$

where $b(x)$ and $c(x)$ are complex-valued smooth functions. If $|\operatorname{Im} b(x)|<1$, then the boundary system $\left\{L, B_{2}\right\}$ satisfies the conditions in Theorem 7.2. If, in particular, $b(x)$ is a real valued function, then $\left\{L, B_{2}\right\}$ satisfies the conditions in Theorem 5.1. Hence $\left\{L, B_{2}\right\}$ is hypoelliptic at the boundary.

Proof. We can choose $\rho=(\ell / 2+1,1)$ and $\sigma=(\ell / 2 k, 1)$. Then $\rho_{0}$ $=\ell / 2+1>\sigma^{0}=\ell / 2 k$. Conditions II. 1, II. $2_{1}$ and II. 3 are obviouly verified. As for Condition II. $2_{2}$, the equalities (5.5), (5.6) now have the forms :

$$
\left\{\begin{array}{l}
{\left[-\frac{d^{2}}{d y^{2}}+\left(x^{2 k}+y^{\ell}\right) \xi^{2}\right] v(y)=0 \quad y>0, \xi \neq 0} \\
{\left[\frac{1}{i} \frac{d}{d y}+b(x) x^{k} \xi\right] v(0)=0}
\end{array}\right.
$$

It follows that

$$
i b(x) x^{k} \xi|v(0)|^{2}+\left[{ }_{0}^{\infty}\left|v^{\prime}(y)\right|^{2} d y+\int_{0}^{\infty}\left(x^{2 k}+y^{\ell}\right) \xi^{2} v(y)^{2} d y=0 .\right.
$$


In case $|\operatorname{Im} b(x)|<1$, we have

$$
\left|\operatorname{Im} b(x) x^{k} \xi\right||v(0)|^{2} \leqq \int_{0}^{\infty}\left|v^{\prime}(y)\right|^{2} d y+x^{2 k} \xi^{2} \int_{2}^{\infty}|v(y)|^{2} d y
$$

Hence we have $v(y) \equiv 0$.

EXAMPLE 2. Consider the boundary value problem:

$$
\begin{aligned}
& L u=\left(D_{y}^{2}+y^{\ell} D_{x}^{2}\right) u(\dot{x}, y)=f(x, y) \quad y>0(\ell=0,1,2, \cdots), \\
&\left.B_{2} u\right|_{y=0}=\left.\left[D_{y}+b(x)\left|D_{x}\right|^{2 /(\ell+2)}+c(x)\right] u\right|_{y=0}=0,
\end{aligned}
$$

where $b(x)$ is a real valued smooth function and $c(x)$ is a complex valued smooth function. We can easily verify that $\left\{L, B_{2}\right\}$ satisfies the conditions in Theorem 5.1.

Remark 1. In the Example 2, we have $\rho=(\ell / 2+1,1)$ and $\sigma=$ $(0,1)$. For the operators of the type $\sigma=(0, \cdots, 0,1, \cdots, 1)$, general boundary value problems have been investigated in [16].

Remark 2. Let $\rho=\left(\rho_{1}, \cdots, \rho_{k}, \rho_{k+1}\right)$ be $\left(\rho_{1}, \cdots, \rho_{k}, 1\right)$ and $\sigma=\left(\sigma_{1}, \cdots\right.$, $\left.\sigma_{k}, \sigma_{k+1}\right)$ be $(0, \cdots, 0,1)$. For such a pair $(\rho, \sigma)$ we consider the Dirichlet boundary value problem

$$
\begin{gathered}
L(x, D) u(x)=f(x) \quad \text { in } x_{N}=y>0 \\
u\left(x^{\prime}, 0\right)=0
\end{gathered}
$$

Suppose that under the Condition II. 1 the Condition II. $2_{1}$ were not satisfied. Then we see, by using the same method as in [3; Theorem 1.1] that the problem (8.5), (8.6) is not hypoelliptic in the upper half plane including the boundary. This shows that the Condition II. $2_{1}$ or II. $2_{2}$ is necessary to obtain the hypoellipticity.

\section{REFERENCES}

[1] Baouendi, M. S., Sur une classe d'opérateur elliptiques dégénérés, Bull. Soc. Math. France, 95 (1967), pp. 45-87.

[2] Fujiwara, D. and Shimakura, N., Sur les problèmes aux limites élliptiques stablement variationnels, J. Math. pures et appl. t. 49 (1970), pp. 1-28.

[ 3 ] Grušin, V. V., On a class of hypoelliptic operators, Math. USSR Sbornik, Vol. 12 (1970), pp. 458-476.

[4] Grušin, V. V., Pseudo-differential operators on $\boldsymbol{R}^{n}$ with bounded symbols, Funkcional. Anal. i Priložen. 4 (1970), 4, pp. 37-50=Functional Anal. Appl. Vol. 4 (1970), pp. 202-212. 
[ 5 ] Grušin, V. V., Hypoelliptic differential equations and pseudo-differential operators with operator-valued symbols, Mat. Sbornik, t. 88 (130), No. 4 (1972), pp. 504521 (Russian).

[6 ] Hörmander, L., Hypoelliptic differential operators, Ann. Inst. Fourier Grenoble, Vol. 11 (1961), pp. 477-492.

[ 7] Hörmander, L., Linear partial differential operators, Springer Verlag, 1964.

[8] Hörmander L., Pseudo-differential operators and hypoelliptic equations, Amer. Math. Soc. Sym. on Singular Intergral Operators, 1966, pp. 138-183.

[ 9 ] Hörmander, L., Hypoelliptic second order differential equations, Acta Math., Vol. 119 (1968), pp. 147-171.

[10] Hörmander, L., On the index of pseudo-differential operators, Elliptische Differentialgeichungen II, Koll. Aug, 1969, in Berlin, Schriftenreihe der Inst. für Math. Deutch. Akad. Wiss. zu Berlin Reihe A, Heft 8.

[11] Kohn, J. J. and Nirenberg, L., An algebra of pseudo-differential operators, Comm. Pure Appl. Math. Vol. 18 (1965), pp. 269-305.

[12] Kumano-go, H. and Taniguchi, K., Oscillatory integrals of symbols of pseudodifferential operators on $\boldsymbol{R}^{n}$ and operators of Fredholm type, Proc. Japan Acad., Vol. 49 (1973), pp. 397-402.

[13] Matsuzawa, T., Sur les équations $u_{t t}+t^{\alpha} u_{x x}=f(\alpha \geqq 0)$, Nagoya Math. J., Vol. 42 (1971), pp. 43-55.

[14] Mizohata, S., Theory of partial differential equations, Oxford, 1973.

[15] Shimakura, N., Problémes aux limites variationnels du type élliptiques, Ann. scient. Éc. Norm. Sup. t. 2 (1969), pp. 255-310.

[16] Višik, M. I. and Grušin, V. V., On a class of higher order degenerate elliptic equations, Math. USSR Sbornik, Vol. 8 (1969), pp. 1-32.

Nagoya University 